
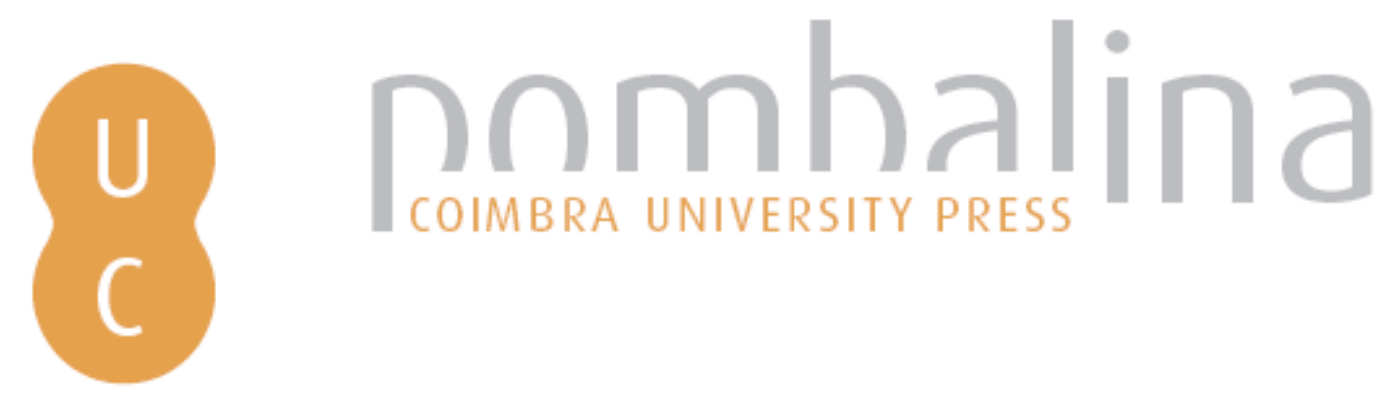

\title{
Utilização de tecnologias SIG e serviços em cloud na avaliação do risco: aplicação à vulnerabilidade estrutural a processos de inundação
}

\author{
Autor(es): \\ Santos, Pedro Pinto dos; Barros, José Leandro; Tavares, Alexandre \\ Oliveira
}

Publicado por: Imprensa da Universidade de Coimbra

URL persistente:

URI:http://hdl.handle.net/10316.2/37075

DOI:

DOI:http://dx.doi.org/10.14195/978-989-26-0983-6_22

Accessed : $\quad$ 26-Apr-2023 06:31:08

A navegação consulta e descarregamento dos títulos inseridos nas Bibliotecas Digitais UC Digitalis, UC Pombalina e UC Impactum, pressupõem a aceitação plena e sem reservas dos Termos e Condições de Uso destas Bibliotecas Digitais, disponíveis em https://digitalis.uc.pt/pt-pt/termos.

Conforme exposto nos referidos Termos e Condições de Uso, o descarregamento de títulos de acesso restrito requer uma licença válida de autorização devendo o utilizador aceder ao(s) documento(s) a partir de um endereço de IP da instituição detentora da supramencionada licença.

Ao utilizador é apenas permitido o descarregamento para uso pessoal, pelo que o emprego do(s) título(s) descarregado(s) para outro fim, designadamente comercial, carece de autorização do respetivo autor ou editor da obra.

Na medida em que todas as obras da UC Digitalis se encontram protegidas pelo Código do Direito de Autor e Direitos Conexos e demais legislação aplicável, toda a cópia, parcial ou total, deste documento, nos casos em que é legalmente admitida, deverá conter ou fazer-se acompanhar por este aviso.

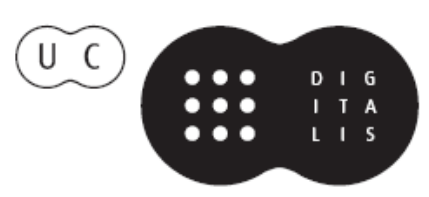




\section{$\forall$ \\ TAS DAS I JORNADAS LUSÓFONAS DE CIÊNCIAS E TECNOLOGIAS DE INFORMAÇÃO GEOGRÁFICA}

Editores

José Gomes dos Santos

Cidália Fonte

Rui Ferreira de Figueiredo

Alberto Cardoso

Gil Gonçalves

José Paulo Almeida

Sara Baptista 


\section{ARTIGO 22}

\section{UTILIZAÇÃO DE TECNOLOGIAS SIG E SERVIÇOS EM CLOUD NA AVALIAÇÃo DO RISCO: APLICAÇÃo À VULNERABILIDADE ESTRUTURAL A PROCESSOS DE INUNDAÇÃo}

SANTOS, Pedro Pinto dos ${ }^{1}$, BARROS, José Leandro ${ }^{2}$ E TAVARES, Alexandre Oliveira ${ }^{3}$

\footnotetext{
${ }^{1}$ Centro de Estudos Sociais da Universidade de Coimbra (Portugal); Colégio de São Jerónimo, 3001-401 Coimbra, Portugal; Tel: +351 239 855570; Fax: +351239 855589; email: pedrosantos@ces.uc.pt 2 Centro de Estudos Sociais da Universidade de Coimbra (Portugal); Colégio de São Jerónimo, 3001-401 Coimbra, Portugal; Tel: +351 239 855570; Fax: +351 239 855589; email: joseleandrobarros@gmail.com 3 Departamento de Ciências da Terra e Centro de Estudos Sociais da Universidade de Coimbra (Portugal); Largo Marquês de Pombal, 3000-272 Coimbra, Portugal; Tel: +351 239 860566; Fax: +351 239 860501; email: atavares@ci.uc.pt
}

\section{RESUMO}

Partindo de dois projetos de investigação científica que têm em comum a realização da avaliação da vulnerabilidade do território aos efeitos de inundação por tsunami e por inundação estuarina agravada por storm surge, o trabalho que se apresenta descreve a metodologia e mais-valias da utilização de tecnologias de informação geográfica nesse processo de avaliação. Concretamente, descreve-se o recurso a soluções de Sistemas de informação geográfica e Sistemas Globais de Navegação por Satélite (Collector for ArcGIS®) a partir de dispositivos móveis (tablet e smartphone) para efetuar levantamento de dados no campo, com apoio de bases de dados partilhadas a partir de serviços em cloud (ArcGIS Online). O trabalho realizado até ao momento demonstra o elevado valor destas ferramentas na aquisição, integração, manipulação e visualização de dados específicos para a avaliação da vulnerabilidade estrutural dos edifícios face aos processos de perigo, bem como o seu 
potencial de integração no planeamento e nas operações de socorro e de emergência de proteção civil.

\title{
PALAVRAS-Chave
}

Serviço SIG em cloud, Trabalho de campo, Vulnerabilidade, Tsunami, Inundação.

\section{USE OF GIS TECHNOLOGIES AND CLOUD SERVICES IN RISK ASSESSMENT: APPLICATION TO STRUCTURAL VULNERABILITY TO FLOODING PROCESSES}

\begin{abstract}
From the starting point of two scientific research projects, that have in common the realization of vulnerability assessments to the effects of flooding from tsunami and river flooding aggravated by storm surge, the presented paper describes the methodology and advantages of using geographic information technologies in the evaluation process. Specifically, we describe the use of solutions of Geographic Information Systems and Global Navigation Satellite Systems (Collector is ArcGIS®) from mobile devices (tablets and smartphones) to perform field data collection, with the support of databases shared through cloud services (ArcGIS Online).

The work conducted until the moment highlights the high value of these tools in the acquisition, integration, manipulation and visualization of specific data to the assessment of structural vulnerability of buildings, in the face of the mentioned hazardous processes, as well as its potential for integration in civil protection emergency planning and relief operations.
\end{abstract}

\section{KEYWORDS}

GIS cloud service, Field work, Vulnerability, Flooding, Tsunami.

\section{INTRODUÇÃO}

A maioria dos domínios científicos apresenta, sob um ou outro prisma, uma dimensão espacial. Nas Ciências do Risco, como esfera do conhecimento marcada por elevada transdisciplinaridade e aplicabilidade ao território, esta dimensão está indelevelmente bem marcada (TAVARES, 2013).

Os avanços das últimas duas décadas na área das tecnologias de informação, ainda que, em alguns casos não inicialmente vocacionados para aplicações geográficas, cedo revelaram o seu potencial para tal utilização, pelas mais-valias em tempo, custo económico e eliminação de 
erros - sobretudo aqueles associados à conversão de dados em formato analógico recolhidos em campo, para formato digital - que proporcionam (LONGLEY et al., 2005). De entre os avanços mais recentes destaca-se o recurso a computação e armazenamento em clouding (XIAOQIANG \& YUEJIN, 2010) e o desenvolvimento de novas aplicações para sistemas operativos, como Android ${ }^{\circledR}$, que correm em dispositivos móveis. De acordo com Naghavi (2012), as principais vantagens dos serviços em cloud consistem na agilidade, acessibilidade aos recursos, partilha e disponibilização, confiabilidade, escalabilidade e facilidade de manutenção.

O acoplamento destas novas ferramentas com outras pré-existentes, como sejam os Sistemas Globais de Navegação por Satélite (GNSS) e os Sistemas de informação geográfica (SIG), permite eficientes e poderosas arquiteturas de aquisição, integração, manipulação e visualização de dados geográficos (YANG et al., 2011; GOODCHILD, 2012).

A disseminação de dispositivos móveis com acesso a georreferenciação fornecida por GNSS, como sejam os smartphones ou tablets, permite soluções de maior versatilidade e menor custo na aquisição e integração de informação geográfica em campo, quando comparadas com soluções baseadas em estações e recetores de GNSS - que recorrem por vezes a aplicações dispendiosas como $\mathrm{ArcPad}$ - nas situações em que a elevada precisão planimétrica e altimétrica não são um requisito.

O trabalho que se apresenta constitui um exemplo de aplicação de tecnologias de informação geográfica (TIG), recorrendo a serviços em cloud, num processo de avaliação da vulnerabilidade estrutural em áreas costeiras, conduzido a partir de dois projetos de investigação científica na área dos riscos naturais. Os sistemas costeiros e estuarinos são ambientes dinâmicos por natureza (CARTER, 1988), mesmo na ausência de processos extremos, sobre os quais a presença e intervenção humana atuam a dois níveis: agravamento da perigosidade - retenção de sedimentos nas albufeiras das barragens, alteração do balanço sedimentar, etc. (e.g. FREIRE et al., 2007) - e da vulnerabilidade, dada a localização face aos processos de perigo (e.g. SANTOS \& KOSHIMURA, 2013).

Após a enunciação dos objetivos e objetos de análise comuns e diferenciadores dos dois projetos de investigação, descreve-se a estrutura 
das matrizes de campo e o recurso à cloud ESRI para a recolha de dados. Por fim, apresentam-se os resultados preliminares obtidos, realçando o papel das TIG no apoio ao trabalho de campo, conducente à concretização do objetivo de caracterização e diferenciação da vulnerabilidade do território. Na sequência dos trabalhos em desenvolvimento posiciona-se a agregação da informação produzida a um nível superior como seja a unidade territorial estatística - subsecção e secção estatística, e freguesia.

\section{OBJETIVOS E OBJETOS DE ANÁlISE}

O trabalho em desenvolvimento está enquadrado em dois projetos de investigação científica em curso: "TSURIMA - Gestão do risco de tsunami para o ordenamento do território e a proteção civil” (RISKAM, 2014), e "MOLINES - Modelação da inundação em estuários: da avaliação da perigosidade à gestão crítica" (LNEC, 2014), financiados pela Fundação para a Ciência e a Tecnologia. O projeto TSURIMA tem por objetivo proceder à modelação numérica da inundação por tsunami, avaliar a vulnerabilidade do território das áreas afetadas e propor estratégias de planeamento de emergência e de ordenamento do território adaptadas aos diferentes cenários de risco. O projeto MOLINES apresenta de um modo geral a mesma sequência metodológica porém, aplicada ao risco de inundação estuarina - componente mareal - agravada por processos de storm surge, i.e., sobrelevação do nível do mar de origem meteorológica, e pelo efeito da drenagem urbana.

A área de estudo do projeto TSURIMA consiste nos concelhos de Albufeira, Figueira da Foz, Setúbal e Vila do Bispo. O projeto MOLINES tem por área de estudo o estuário do Tejo desde os concelhos de Vila Franca de Xira e Benavente, a montante, até aos concelhos de Oeiras e Almada, a jusante.

\section{METODOLOGIA}

A metodologia que serve de base ao presente artigo pode ser dividida em duas fases distintas: a primeira foi realizada essencialmente 
em ambiente desktop e a segunda centrou-se na realização de trabalho de campo. O processo metodológico aqui apresentado foi aplicado nos projetos MOLINES e TSURIMA. Ambos os projetos pretendem avaliar o edificado potencialmente afetado por inundações. No caso do MOLINES pretende-se avaliar os processos de inundação em margens estuarinas, incluindo os processos de sobrelevação meteorológica conjugados com o efeito da maré, e a drenagem urbana. No que concerne ao TSURIMA pretende-se avaliar os edifícios potencialmente afetados por inundação devido a tsunami, tendo por base a modelação numérica do tsunami de 1 de Novembro de 1755.

Para tal foi necessário recolher e selecionar com base na bibliografia e na opinião de peritos um conjunto de parâmetros intrínsecos ao edifício e à sua área envolvente que auxiliassem na avaliação da sua vulnerabilidade. Deste processo resultou a construção de matrizes de avaliação de campo para os dois projetos, atendendo às especificidades de cada um. Devido à diferença na escala de análise, a área envolvente considerada no projeto TSURIMA agrega-se à área de inundação e no projeto MOLINES à subsecção estatística. Concretamente, a matriz de avaliação de campo para os edifícios apresenta um total de 11 parâmetros no projeto MOLINES e um total de 13 parâmetros no projeto TSURIMA, relacionados com estrutura, função, ocupação e flutuação ocupacional do edifício e suas unidades de utilização, ou alojamentos (Tabela 1). A área envolvente é descrita segundo 5 parâmetros tanto na matriz do projeto MOLINES, como na matriz do projeto TSURIMA (Tabela 2). As diferenças advêm do facto de que existem parâmetros específicos para a inundação por tsunami que não têm relevância para a inundação estuarina, relacionados com a diferente hidrodinâmica de cada processo. Nestas matrizes descreve-se a ocupação do solo predominante na área envolvente - secção ou subsecção estatística -, os elementos sensíveis e de capacidade de suporte existentes (lares de terceira idade, centros de saúde, quartéis de bombeiros, etc.) e as características do fabric urbano, e de elementos de proteção natural e estrutural. 
Tabela 1 - Parâmetros de avaliação dos edifícios, propostos nos projectos TSURIMA e MOLINES

\begin{tabular}{|l|c|c|}
\cline { 2 - 3 } \multicolumn{1}{c|}{} & TSURIMA & MOLINES \\
\hline $\begin{array}{l}\text { Forma de desenvolvimento do } \\
\text { edificado em planta }\end{array}$ & $\times$ & $\times$ \\
\hline $\begin{array}{l}\text { Altura emersa do edifício em relação } \\
\text { à inundação/onda }\end{array}$ & $\times$ & $\times$ \\
\hline $\mathrm{N}^{\circ}$ de pisos & $\times$ & $\times$ \\
\hline Material de construção & $\times$ & $\times$ \\
\hline Ano de construção & $\times$ & $\times$ \\
\hline Estado de conservação & $\times$ & $\times$ \\
\hline Hidrodinâmica r/c & $\times$ & $\times$ \\
\hline Existência de pisos subterrâneos & $\times$ & $\times$ \\
\hline Tipo de fundação & $\times$ & $\times$ \\
\hline Forma de ocupação & $\times$ & $\times$ \\
\hline $\mathrm{N}^{\circ}$ de unidades de utilização & $\times$ & \\
\hline $\begin{array}{l}\mathrm{N}^{\circ} \text { médio diário de pessoas presentes } \\
\text { ou visitantes por unidade de } \\
\text { utilização }\end{array}$ & $\times$ & \\
\hline Flutuação de ocupação & & $\times$ \\
\hline Permanência à exposição & & \\
\hline
\end{tabular}

Tabela 2 - Parâmetros de avaliação da área envolvente, propostos nos projectos TSURIMA e MOLINES

\begin{tabular}{|c|c|c|}
\hline & TSURIMA & MOLINES \\
\hline Morfologia da área inundada & $x$ & \\
\hline $\begin{array}{l}\text { Consolidação dos materiais } \\
\text { geológicos }\end{array}$ & $x$ & \\
\hline Declive médio & $x$ & \\
\hline Distância à linha de costa & $x$ & \\
\hline Uso e ocupação do solo & $x$ & $x$ \\
\hline Capacidade de suporte & & $x$ \\
\hline $\begin{array}{l}\text { Presença de objectos móveis entre a } \\
\text { linha de costa e os edifícios }\end{array}$ & & $x$ \\
\hline $\begin{array}{l}\text { Presença de elementos de proteção } \\
\text { intermédios entre a linha de costa e } \\
\text { os edifícios em análise }\end{array}$ & & $x$ \\
\hline $\begin{array}{l}\text { Qualificação urbana e capacidade de } \\
\text { fluxo }\end{array}$ & & $x$ \\
\hline
\end{tabular}


A matriz apresenta um conjunto de atributos intrínsecos a cada parâmetro e atribui a cada atributo um determinado valor que traduz a maior ou menor contribuição do mesmo para a vulnerabilidade do edifício analisado.

Após a definição da matriz foi necessária a criação de uma base de dados que serviu de ponto de partida para a criação de fichas de campo em formato SIG o que permitiu o levantamento in loco dos vários atributos da matriz. Esta base de dados foi realizada recorrendo ao software $\operatorname{ArcGIS}{ }^{\circledR}$ versão 10.2 , tendo sido criada uma feature associada à base de dados. Os serviços fornecidos por cloud computing constituem uma das principais inovações na área das TIG (NAGHAVI, 2012). Fazendo uso deste tipo de serviços, recorreu-se ao portal ArcGIS Online com o intuito de fazer o upload na nossa base de dados para a cloud da ESRI para que ela estivesse disponível online para posterior preenchimento e upload a partir do campo (Figura 1 e 2). Como se observa, neste momento o conteúdo do mapa consiste numa geodatabase com o nome "SeixalFW", a qual tem uma feature de nome "Edificios", e uma imagem ou basemap fornecida por serviço WMS (neste caso, World Imagery).

Após a criação da base de dados e o seu upload na cloud foi possível passar à fase de elaboração do trabalho de campo. No terreno foi utilizado um tablet Samsung ${ }^{\circledR}$ com tecnologia Android ${ }^{\circledR}$ e com a aplicação Collector for ArcGIS® instalada no dispositivo móvel. Esta aplicação permite realizar o download da base de dados criada em ambiente desktop, de modo a se proceder ao preenchimento in loco dos diferentes parâmetros dos edifícios potencialmente afetados. Adicionalmente, a aplicação utilizada permite a associação de fotografias a cada elemento da base de dados, bem como a recolha de outras observações exteriores à mesma, com base na localização providenciada pelo Sistema Global de Navegação por Satélite, no caso, o Global Positioning System (GPS). Após a realização desta tarefa os dados recolhidos no terreno são guardados na cloud para posterior utilização no ambiente desktop.

Com o levantamento de campo finalizado e o tratamento da base de dados terminada, são realizados um conjunto de outputs que se traduzem na produção de cartografia diversa que apresenta os diversos parâmetros 
da matriz recolhidos e que auxiliam na análise e avaliação do edificado potencialmente afetado.

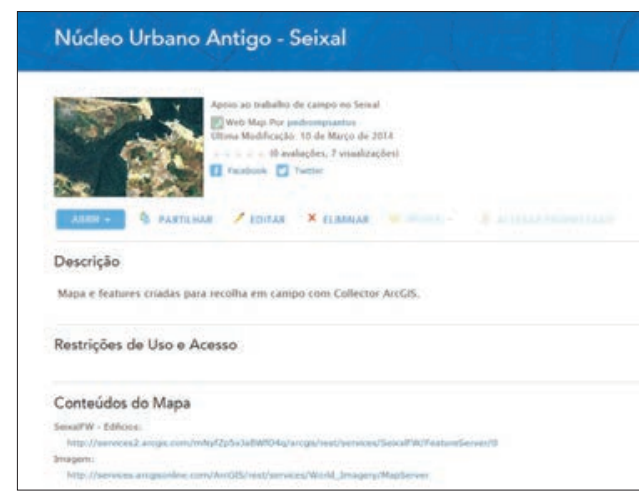

Figura 1 - Resumo do mapa criado em ambiente cloud no ArcGIS Online para recolha de dados no campo

A disponibilização dos dados na cloud permite não só a atualização dos dados pelos vários intervenientes no trabalho de campo, como também a partilha para os diferentes stakebolders. Esta capacidade permite o desenvolvimento de um trabalho integrado e corporativo com os diferentes atores locais, permitindo a inserção de novos dados e atualização dos já existentes. Esta potencialidade de alojamento e partilha através da cloud permite o envolvimento de todas as partes no processo de análise e avaliação da vulnerabilidade e no desenvolvimento e adoção de medidas de mitigação do risco.

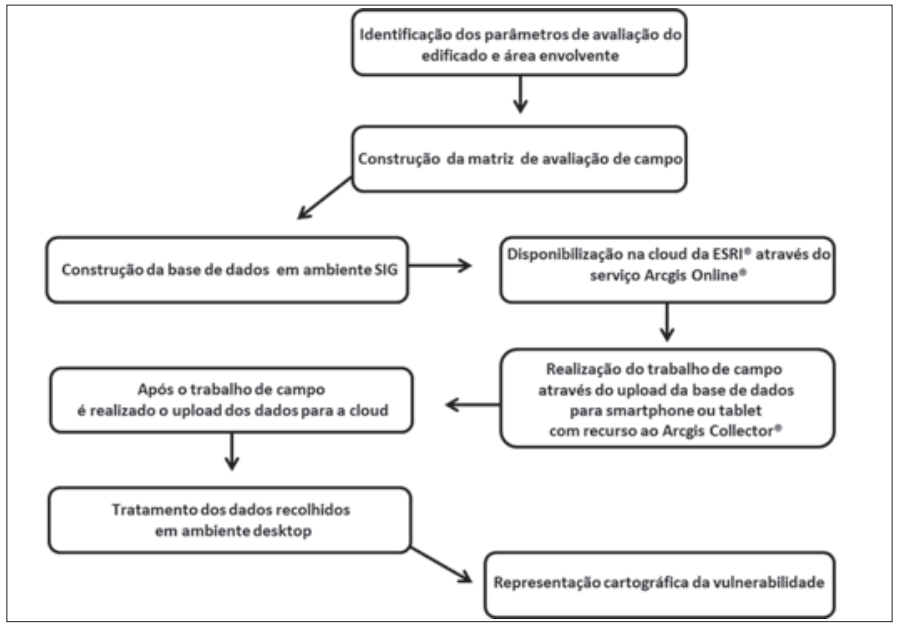

Figura 2 - Representação gráfica da metodologia aplicada na recolha de dados do trabalho de campo 


\section{RESULTADOS}

A tipologia do parque habitacional consiste numa característica relevante no âmbito dos dois projetos de investigação indicados. Com efeito, os edifícios residenciais fazem parte dos elementos expostos mais frequente e severamente afetados diretamente por tsunami e inundação estuarina. Tratam-se igualmente de elementos vulneráveis em termos de valor económico por unidade de superfície (DE MOEL \& AERTS, 2011). Ademais, os edifícios redirecionam o seu efeito para a dimensão das perdas pessoais (morte, ferimento, desalojamento e evacuação). As matrizes de avaliação de campo expressam essa relevância em ambos os projetos, com ligeiras diferenças ao nível dos campos e atributos. A Figura 3 ilustra um output preliminar produzido no âmbito do projeto MOLINES que combina a altura dos edifícios com a hidrodinâmica do piso térreo, em duas subsecções estatísticas da área do Núcleo Urbano Antigo do Seixal. Os edifícios com um único piso - não considerando os restantes parâmetros - são teoricamente os mais vulneráveis porquanto não permitem quer a evacuação e permanência de pessoas quer a mobilização do recheio das habitações para outros pisos dentro do mesmo edifício. O cruzamento dos diversos parâmetros irá permitir uma adequada caracterização da vulnerabilidade do edificado.

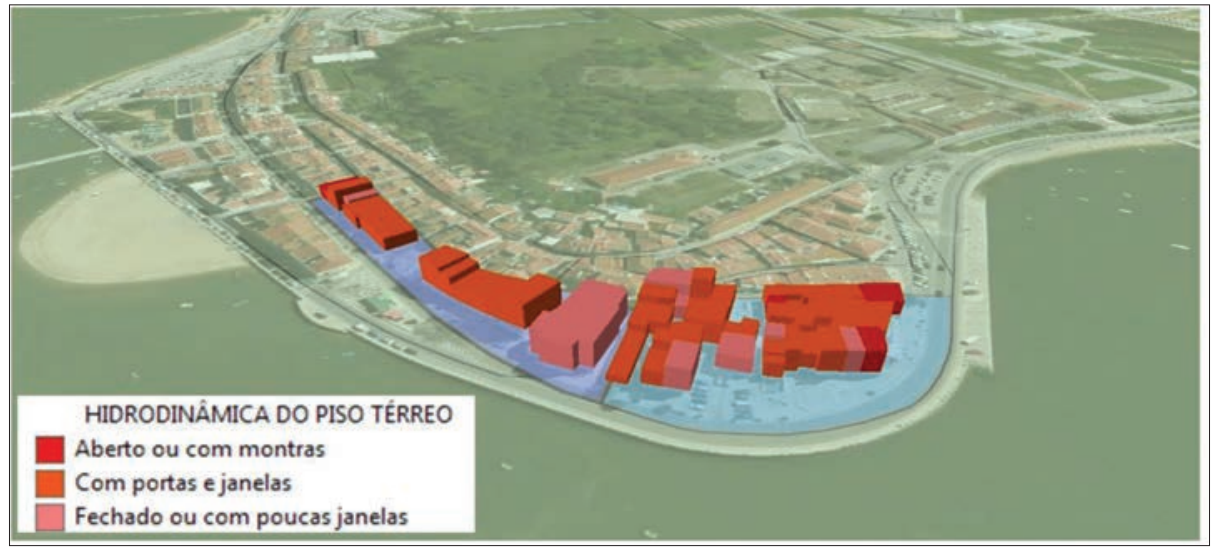

Figura 3 - Representação da altura do edificado e da hidrodinâmica do piso térreo a partir de dados recolhidos com a aplicação Collector for ArcGIS® instalada em dispositivo móvel (Projeto MOLINES) 
Várias metodologias abordam a temática da vulnerabilidade de edifícios face a tsunamis (PAPATHOMA et al., 2003; PAPATHOMA \& DOMINEYHOWES, 2003; DOMINEY-HOWES \& PAPATHOMA, 2007; DALL'OSSO et al., 2009; KAPLAN et al., 2009; DALL'OSSO et al., 2010; ANPC, 2010; OMIRA et al., 2010; LEONE et al., 2011; RIBEIRO et al., 2011; ISMAIL et al., 2012; BARROS et al., 2013; EMÍDIO et al., 2013), recorrendo a um conjunto de parâmetros intrínsecos aos edifícios, recolhidos com base em análise de imagens de satélite e avaliação in loco das características dos edifícios. Estas metodologias consideram um conjunto de parâmetros mais limitado em comparação com a metodologia preconizada pelo projeto TSURIMA, na qual se condensaram e acrescentaram parâmetros não considerados anteriormente, resultando num total de 13 parâmetros diretamente relacionados com o edificado. A Figura 3 dá a conhecer a expressão cartográfica de um desses parâmetros que é o "estado de conservação". O presente levantamento foi efetuado na praia da Salema, Vila do Bispo, onde foram identificados um total de 44 edifícios potencialmente afetados.

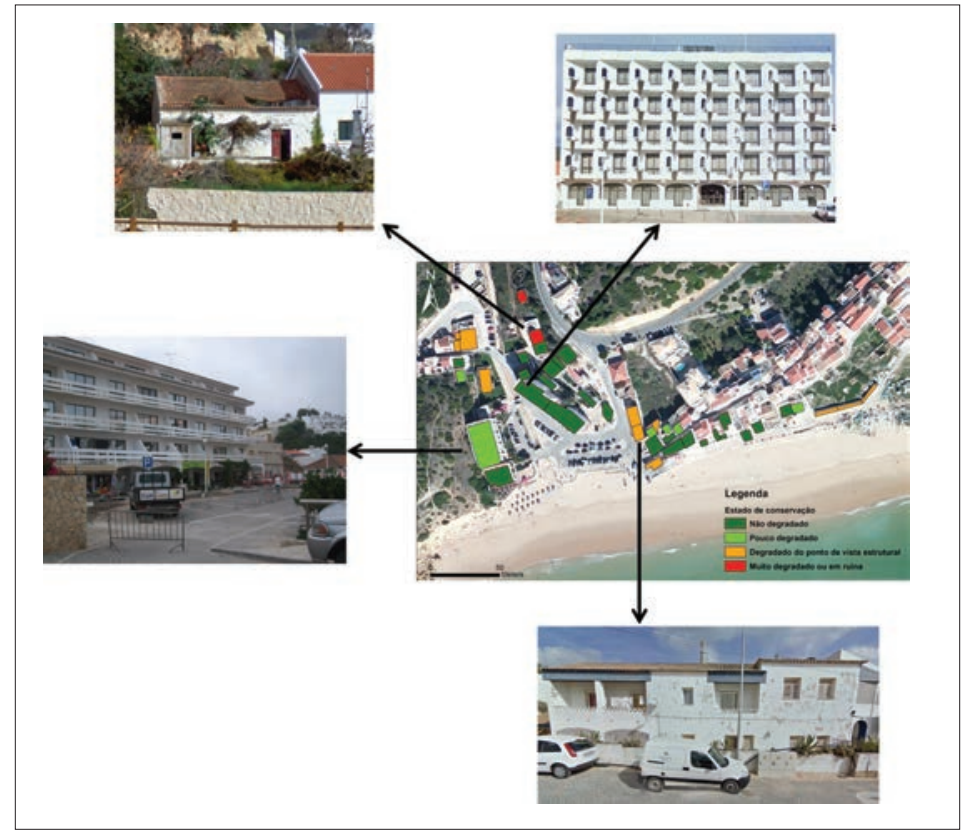

Figura 3 - Representação do estado de conservação dos edifícios a partir de dados recolhidos com a aplicação Collector for ArcGIS® instalada em dispositivo móvel (Projeto TSURIMA) 
A análise e avaliação in loco dos diferentes edifícios e dos parâmetros intrínsecos a cada um deles torna-se fundamental para o processamento da avaliação da vulnerabilidade estrutural dos edifícios face a tsunamis. No que concerne especificamente ao parâmetro apresentado na Figura 3 a sua análise foi realizada com base em quatro classes (não degradado; pouco degradado; degradado do ponto de vista estrutural; muito degradado ou em ruína). Segundo Dall'Osso et al., (2009) a introdução deste parâmetro na análise é importante pois permite identificar os edifícios em pior estado de conservação, ou seja, mais suscetíveis a sofrem danos elevados face a tsunamis, nomeadamente se apresentarem graves deformações ou falhas estruturais importantes. No que respeita à área da praia da Salema pode concluir-se que 57\% dos edifícios analisados são identificados como "não degradado", 25\% como "degradado do ponto de vista estrutural", 14\% como "pouco degradado" e 4\% como "muito degradado ou em ruina”.

\section{CONCLUSÕES}

A utilização de tecnologias de informação geográfica descrita a partir do trabalho desenvolvido nos projetos TSURIMA e MOLINES permite concluir sobre as vantagens do recurso a serviços SIG em cloud no apoio ao trabalho de campo. A mais-valia destes serviços obtém-se pelo seu acoplamento a aplicações SIG, tais como o Collector for ArcGIS®, que correm em dispositivos móveis. Posteriormente, evidencia-se a facilidade de partilha de dados entre os diferentes utilizadores da organização e, subsequentemente, caso entendível, com a comunidade geográfica em geral através do serviço ArcGIS Online. Finalmente, realça-se a futura integração dos dados recolhidos com outras fontes de informação relacionadas com a caracterização da vulnerabilidade, em ambiente SIG. Neste aspeto, merece referência a integração com os dados censitários que, desde o XIV Recenseamento Geral da População, de 2001, são disponibilizados com associação à Base Geográfica de Referenciação da Informação (BGRI) ao nível da subsecção estatística, num total de 128 variáveis relativas à demografia, educação, edificado, condições dos alojamentos, composição 
do agregado familiar, emprego e mobilidade.

O cruzamento dos dados recolhidos ao nível do edifício com os dados caracterizadores do território envolvente permite uma avaliação pormenorizada da vulnerabilidade aos processos de perigo. Neste sentido, as ferramentas utilizadas têm um potencial elevado de utilização no apoio às operações de socorro e de planeamento de emergência de proteção civil.

\section{AgRAdeCIMENTOS}

Este trabalho é financiado por Fundos FEDER através do Programa Operacional Factores de Competitividade - COMPETE e por Fundos Nacionais através da FCT - Fundação para a Ciência e a Tecnologia no âmbito dos projetos "TSURIMA - Gestão do risco de tsunami para o ordenamento do território e a proteção civil» (PTDC/CS-GEO/118992/2010), e "MOLINES - Modelação da inundação em estuários: da avaliação da perigosidade à gestão crítica”»(PTDC/AAG-MAA/2811/2012).

\section{BIBLIOGRAFIA}

ANPC (2010) - "Estudo do Risco Sísmico e de Tsunamis do Algarve (ERSTA)". Autoridade de Protecção Civil. 85 pp. Disponível online no endereço url: http://www.prociv.pt/Pages/detalhe4.aspx?IDitem=58 (acedido em 1 fevereiro, 2014).

CARTER, Richard W. G. (1988) - Coastal environments. Academic Press, Londres, $617 \mathrm{p}$.

de MOEL, Hans \& AERTS, Jeroen (2011) - "Effect of uncertainty in land use, damage models and inundation depth on flood damage estimates". Natural Hazards, Vol. 58, 407-425. Disponível online no endereço url: http://link. springer.com/article/10.1007\%2Fs11069-010-9675-6\#page-1 (acedido em 3 fevereiro, 2014).

BARROS, José Leandro et al. (2013) - "Metodologias de avaliação da vulnerabilidade ao risco de tsunami: aplicação ao sector costeiro Cova Gala - Leirosa; Figueira da Foz". Pinto Correia, T.; Henriques, V.; Julião, R.P. (Eds.), IX Congresso da Geografia Portuguesa - Geografia: Espaço, Natureza, Sociedade e Ciência, p. 839-845, Evora, Portugal, 28-30 Novembro, 2013. ISBN:978972-99436-6-9. Disponível online no endereço url: https://sites.google. com/a/campus.ul.pt/tsurima/publications (acedido em 1 fevereiro, 2014). 
DALL'OSSO, Filippo et al. (2009) - "Applying and validating the PTVA-3 Model at the Aeolian Islands, Italy: assessment of the vulnerability of buildings to tsunamis". Nat. Hazards Earth Syst. Sci., 10, 1547-1562, doi:10.5194/ nhess-10-1547-2010. Disponível online no endereço url: http://www.nat-hazards-earth-syst-sci.net/10/1547/2010/nhess-10-1547-2010.html (acedido em 5 fevereiro, 2014).

DALL'OSSO, Filippo et al. (2010) - "A novel approach (the CRATER method) for assessing tsunami vulnerability at the regional scale using ASTER imagery". Italian Journal of Remote Sensing 42(2): 55-74. Disponível online no endereço url: http://www.aitjournal.com/articleView.aspx?ID=164 (acedido em 5 fevereiro, 2014).

DOMINEY-HOWES, Dale \& PAPATHOMA, Maria (2007) - "Validating a tsunami vulnerability assessment model (the PTVA model) using field data from the 2004 Indian Ocean tsunami”. Natural Hazards, 40, 113-136. Disponível online no endereço url: http://link.springer.com/article/10.1007/s11069006-0007-9\# (acedido em 1 fevereiro, 2014).

EMÍDIO, António et al. (2013) - "Aplicação da metodologia PTVA-3 na avaliação da vulnerabilidade do centro urbano da cidade de Setúbal em caso de tsunami”. Pinto Correia, T.; Henriques, V.; Julião, R.P. (Eds.), IX Congresso da Geografia Portuguesa - Geografia: Espaço, Natureza, Sociedade e Ciência, p. 833-838, Evora, Portugal, 28-30 Novembro, 2013. ISBN:978-972-99436-69. Disponível online no endereço url: https://sites.google.com/a/campus. ul.pt/tsurima/publications (acedido em 3 fevereiro, 2014).

FREIRE, Paula, TABORDA, Rui \& SILVA, Ana M. (2007) - "Sedimentary Characterization of Tagus Estuarine Beaches (Portugal). A contribution to the sediment budget assessment". Journal of Soils and Sediments, Vol. 7, $\mathrm{N}^{\circ}$ 5, 296-302. Disponível online no endereço url: http://dx.doi.org/10.1065/ jss2007.08.243 (acedido em 5 fevereiro, 2014).

GOODCHILD, Michael F. (2012) - "The future of Digital Earth". Annals of GIS, Vol. 18, $\mathrm{N}^{\mathrm{o}} 2$ 2, 93-98. Disponível online no endereço url: http://dx.doi. org/10.1080/19475683.2012.668561 (acedido em 28 janeiro, 2014).

ISMAIL, Hadibah et al. (2012) - "A 3-tier tsunami vulnerability assessment technique for the nort-west coast of Peninsular Malaysia”. Natural Hazards, 63, 549-573. Disponível online no endereço url: http://link.springer.com/ article/10.1007/s11069-012-0166-9\# (acedido em 1 fevereiro, 2014).

KAPLAN, Marcus et al. (2009) - "Vulnerability assessment and protective effects of coastal vegetation during the 2004 Tsunami in Sri Lanka". Nat. Hazards Earth Syst. Sci., 9, 1479-1494, doi:10.5194/nhess-9-1479-2009. Disponível online no endereço url: http://www.nat-hazards-earth-syst-sci.net/9/1479/2009/ nhess-9-1479-2009.html (acedido em 5 fevereiro, 2014).

NAGHAVI, Mohammad (2012) - "Cloud computing as an innovation in GIS \& SDI: Methodologies, services, issues and deployment techniques”. Journal of 
Geographic Information System, 4, 6, 597-607, Disponível online no endereço url: http://www.scirp.org/journal/PaperInformation.aspx?PaperID=26325\# . U7GHevldUa4 (acedido em 27 junho, 2014).

LEONE, Frédéric et al. (2011) - "A spatial analysis of the December 26th, 2004 tsunami-induced damages: lessons learned for a better risk assessment integrating buildings vulnerability". Appl Geogr 31(1):363-375. Disponível online no endereço url: http://www.sciencedirect.com/science/article/pii/ S0143622810000834 (acedido em 5 fevereiro, 2014).

LNEC (2014) Página eletrónica do projeto MOLINES disponível online no endereço url: http://www.lnec.pt/organizacao/dha/nec/estudos_id/Molines (acedido em 4 março, 2014).

LONGLEY, Paul A., et al. (2005) - "Geographic Information Systems and Science". $2^{\text {nd }}$ edition, John Wiley \& Sons, LTD, West Sussex, 512 p.

OMIRA, Rachid et al. (2010) - "Tsunami vulnerability assessment of CasablancaMorocco using numerical modelling and GIS tools". Natural Hazards, 54, 1, 75-95. Disponível online no endereço url: http://link.springer.com/article/10.1007/s11069-009-9454-4 (acedido em 2 fevereiro, 2014)

PAPATHOMA, Maria, et al. (2003) - "Assessing tsunami vulnerability, an example from Herakleio”. Crete, Nat. Hazards Earth Syst. Sci., 3, 377-389, doi:10.5194/ nhess-3-377-2003. Disponível online no endereço url: http://www.nat-hazards-earth-syst-sci.net/3/377/2003/nhess-3-377-2003.html (acedido em 1 fevereiro, 2014).

PAPATHOMA, Maria \& DOMINEY-HOWES, Dale (2003) - "Tsunami vulnerability assessment and its implications for coastal hazard analysis and disaster management planning, Gulf of Corinth, Greece”. Nat. Hazards Earth Syst. Sci., 3, 733-747, doi:10.5194/nhess-3-733-2003. Disponível online no endereço url: http://www.nat-hazards-earth-syst-sci.net/3/733/2003/nhess-3-733-2003. html (acedido em 1 fevereiro, 2014).

RIBEIRO, João et al. (2011) - "High resolution tsunami modelling for the evaluation of potential risk areas in Setúbal (Portugal)”. Nat. Hazards Earth Syst. Sci., 11, 2371-2380, doi:10.5194/nhess-11-2371-2011. Disponível online no endereço url: http://www.nat-hazards-earth-syst-sci.net/11/2371/2011/ nhess-11-2371-2011.html (acedido em 1 fevereiro, 2014).

RISKAM (2014) Página eletrónica do projeto TSURIMA disponível online no endereço url: https://sites.google.com/a/campus.ul.pt/tsurima/ (acedido em 5 março, 2014).

SANTOS, Ângela \& KOSHIMURA, Shunichi (2013) - "Tsunami hazards at Setúbal urban area considering the 1755 Lisbon Tsunami”. Pinto Correia, T.; Henriques, V.; Julião, R.P. (Eds.), IX Congresso da Geografia Portuguesa - Geografia: Espaço, Natureza, Sociedade e Ciência, p. 930-935, Évora, Portugal, 28-30 Novembro, 2013. ISBN:978-972-99436-6-9. Disponível online no endereço 
url: https://sites.google.com/a/campus.ul.pt/tsurima/publications (acedido em 4 fevereiro, 2014)

TAVARES, Alexandre Oliveira (2013) - "Referenciais e modelos de governação dos riscos”, in LOURENÇO, Luciano Fernandes \& MATEUS, Manuel Alberto (coord.): Riscos naturais, antrópicos e mistos. Homenagem ao Professor Doutor Fernando Rebelo, Coimbra, Departamento de Geografia, Faculdade de Letras, Universidade de Coimbra, 63-80. Disponível online no endereço url: http://www.uc.pt/fluc/depgeo/Publicacoes/livro_homenagem_FRebelo/063_080 (acedido em 24 janeiro, 2014).

XIAOQIANG, Yang \& YUEJIN, Deng (2010) - "Exploration of cloud computing technologies for geographic information services. In Proceedings of the $18^{\text {th }}$ International Conference on Geoinformatics, Beijing, China. Disponível online no endereço url: http://ieeexplore.ieee.org/xpl/articleDetails. jsp?arnumber=5567515 (acedido em 26 junho, 2014).

YANG, Chaowei et al. (2011) - "Spatial cloud computing: how can the geospatial sciences use and help shape cloud computing?" International Journal of Digital Earth, Vol. 4, N. ${ }^{\circ} 4,305-329$. Disponível online no endereço url: http://dx.doi.org/10.1080/17538947.2011.587547 (acedido em 6 março, 2014). 
Série Documentos

Imprensa da Universidade de Coimbra

Coimbra University Press

2015

- U M

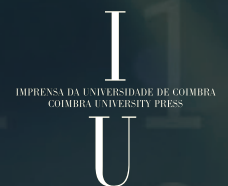

